\title{
Bioartificial Liver Device Based on Induced Pluripotent Stem Cell-Derived Hepatocytes
}

\author{
Songyang Ren ${ }^{1}$, Joseph Ignatius Irudayam ${ }^{1}$, Deisy Contreras ${ }^{1}$, Dhruv Sareen ${ }^{1}$, Dodanim Talavera-Adame ${ }^{1,2}$, Clive N Svendsen $^{1,3}$ and \\ Vaithilingaraja Arumugaswami ${ }^{1-3 *}$
}

${ }^{1}$ Board of Governors Regenerative Medicine Institute, Cedars-Sinai Medical Center, Los Angeles, USA

${ }^{2}$ Department of Surgery, Cedars-Sinai Medical Center, Los Angeles, USA

${ }^{3}$ David Geffen School of Medicine, University of California at Los Angeles, Los Angeles, USA

\begin{abstract}
Decompensated liver disorders require liver transplantation. However, the donor organ shortage is a limiting factor. Harnessing the power of human induced pluripotent stem cell (iPSC) technology in combination with hollow fiber-based bioartificial liver (BAL) device can be beneficial to patients with liver failure. Our goal is to develop a BAL module comprised of iPSC-derived hepatocytes (iHeps) arrayed on the extracapillary space (ECS) of hollow fiber membranous capillaries that allow the flow of blood through the intracapillary space (ICS), thus mimicking the tissue microarchitecture. For the proof-of-concept in vitro study, a cartridge having semipermeable polysulfone membrane fibers was used as an artificial liver device. As a source for human liver cells, we derived metabolically active hepatocytes from iPSCs. The iHeps on microcarrier beads were loaded into the ECS of a hollow fiber bioreactor cartridge and cultured using a closed-circuit continuous flow system. The iHeps secreted human albumin, prothrombin, and apolipoprotein B into the hollow fiber ICS media, and the continuous flow system also improved maturation of iHeps. In conclusion, the iPSC-hepatocytes in the bioartificial liver device maintained the secretory function and exhibited cell maturation. The iPSC-hepatocyte BAL has the potential to be further developed as a liver support device for the treatment of decompensated liver diseases.
\end{abstract}

Keywords: Induced pluripotent stem cell; iPSC; Hepatocytes derived from iPSC; Bioartificial liver device; Hollow fiber bioreactors; iPSC differentiation; Endoderm

\section{Introduction}

Liver, the largest metabolic organ, is involved in performing several vital functions including metabolism of carbohydrates, lipids, proteins, hormones, and xenobiotics, as well as the secretion of plasma proteins and bile. These functions are carried out by the major liver parenchymal cells, hepatocytes. Injury to hepatocytes due to viral, alcoholic and drug-related causes can disrupt metabolic homeostasis. According to the Center for Disease Control, an estimated 500 million people worldwide are infected with viral hepatitis, and an estimated 1 million people die annually from related causes [1]. Many other autoimmune and toxic exposures can also lead to end-stage liver disease: $35 \%$ of heavy drinkers develop acute alcoholic hepatitis, and drugs as common as acetaminophen can lead to acute liver injury. Patients with acute liver failure (ALF) have a mortality rate of over $80 \%[2,3]$. Both acute and chronic liver disease can lead to liver failure and require costly liver transplantation; which rely on the scarce supply of available organs.

Alternative approaches to liver organ transplantation such as nonbiological and biological liver support systems have been attempted [4-16]. Non-biological systems include blood transfusion, plasma exchange, hemodialysis (to remove $<15 \mathrm{kDa}$ factors), hemofiltration, and hemoadsorption using non-biologic adsorbents (charcoal or resin). A biological liver support system is comprised of ex vivo liver perfusion (livers from human, non-human primate and pig species) and a hepatocyte system (human or pig hepatocytes loaded onto bioreactor devices). The extracorporeal liver assist device (ELAD), HepatAssist liver support system, Molecular Adsorbent Recirculating System (MARS) and The Prometheus device have been in clinical evaluation $[5,11,17,18]$. To date, non-biological liver support systems have been shown to be less effective and, while intuitively more promising, no truly effective biological device has yet to be developed for human treatment purposes [5,18-22].
In general, a biological liver support system is comprised of several distinct components: (a) a cell source such as human hepatocytes (cadaveric primary hepatocytes or hepatic cancer cells from HepG2 cell line) or xeno-hepatocytes (pig or dog), (b) a bioreactor to house the cells, and (c) a perfusion system for blood or plasma [11,19,23]. Hollow fiber capillary bioreactors loaded with hepatocytes of pig origin or HepG2 sub clone C3A cells in the extracapillary space (ECS) for detoxification and plasma protein synthesis purposes have been under clinical investigation [24-27]. The plasma from a liver failure patient is perfused through the intracapillary space (ICS) of bioreactors so it could be cleared of toxic metabolites by ECS hepatocytes via bidirectional mass transport at the membrane (pore size of 0.1 to 0.2 $\mu \mathrm{m})$ in the semipermeable hollow fiber. In clinical trials, the use of extracorporeal BAL systems have resulted in some improvement in the patient's neurological score, blood chemistry, and prothrombin time $[11,17,26,27]$.

A major current limitation to this approach is the quality control and risk of using either pig hepatocytes or human cancer cells [23,28]. Animal cells confer a risk of zoonotic disease transmission, making human cells more acceptable [29]. Unfortunately, a major bottleneck on such a device is the scarce availability of human hepatocytes. HepG2based human tumor cells pose a theoretical risk for transmission of

*Corresponding author: Vaithilingaraja Arumugaswami, 8700 Beverly Blvd AHSP, A8416, Los Angeles, CA 90048, USA, Tel: +1 (310) 248 8584; Fax: +1 (310) 248 8066; E-mail: arumugaswami@cshs.org

Received December 25, 2014; Accepted February 01, 2015; Published February 03, 2015

Citation: Ren S, Ignatius Irudayam J, Contreras D, Sareen D, Talavera-Adame D, et al. (2015) Bioartificial Liver Device Based on Induced Pluripotent Stem Cell-Derived Hepatocytes. J Stem Cell Res Ther 5: 263. doi:10.4172/2157-7633.1000263

Copyright: (c) 2015 Ren S, et al. This is an open-access article distributed unde the terms of the Creative Commons Attribution License, which permits unrestricted use, distribution, and reproduction in any medium, provided the original author and source are credited. 
malignancy through a leaky membrane and paracrine factors secreted into the circulation [23]. Indeed, a previous study has shown that HepG2 conditioned media can induce transformation of normal cells [30]. Recent evidence suggests oncogenic microRNAs and proteins are secreted by tumor cells via exosomes [31,32] and could pose serious health risks. Metabolically active human hepatocytes derived from a well-characterized induced pluripotent stem cell (iPSC) line could offer a tremendous advantage over the current BAL cell sources. Either autologous or allogeneic iPSC-hepatocytes (iHeps) can be used. Our overall goal is to develop a bioartificial liver device based on combining existing iPSC technology and hollow fiber-based bioreactors for the treatment of liver failure. The iHep-BAL can substitute for the metabolic and detoxification functions of the liver. In this study, we describe the design and development of a closed-circuit bioartificial liver module based on human iPSC-hepatocyte components and in vitro functional assessment of a prototype iHep-BAL.

\section{Materials and Methods}

\section{Cells}

The human iPSC line 83i-CTRL was obtained from Cedars-Sinai Medical Center iPSC core facility and was described previously [33]. The iPSC 83i-CTRL line was established by reprogramming normal human fibroblasts using non-integrating expression vectors carrying the OCT4, SOX2, KLF4 and L-MYC genes. The human embryonic stem cell (hESC) line WA09 (H9) was obtained from WiCell Research Institute, USA. The iPSCs and hESCs were cultured using serumfree chemically defined media, mTeSR1 (STEMCELL Technologies, Canada), with a daily media change regimen at $37^{\circ} \mathrm{C}$ incubator with $5 \% \mathrm{CO}_{2}$. The human liver cancer cell line HepG2 and Huh-7.5.1 was maintained on complete Dulbecco's modified Eagle's medium (DMEM) (Fisher Scientific). Complete DMEM was supplemented with $10 \%$ fetal bovine serum (FBS), $10 \mathrm{mM}$ Hepes, $10 \mathrm{mM}$ nonessential amino acids, penicillin (100 units $/ \mathrm{ml})$, streptomycin $(100 \mathrm{mg} / \mathrm{ml})$, and $2 \mathrm{mM}$ L-glutamine (Life Technologies).

\section{In vitro differentiation of human iPSCs into hepatic lineage cells}

For in vitro differentiation, the iPSCs were single cell plated on matrigel in a 6 well-plate, cultured at $37^{\circ} \mathrm{C}$ in $5 \% \mathrm{CO}_{2}$, and subjected to a modified three-week hepatic differentiation protocol [34]. The differentiation steps consisted of three phases: endoderm induction (days 1-5), hepatic specification (days 6-15) and hepatic maturation (days 16-21). The cytokines were purchased from Peprotech Inc., (Rocky Hill, NJ) unless otherwise mentioned. The cells were differentiated to endoderm for 5 days using IMDM/F12 or RPMI media (Life Technologies) supplemented with Wnt 3A (40 ng/ml, R and D Systems) and Activin A $(100 \mathrm{ng} / \mathrm{ml})$ for the first day and then treated with Activin A, VEGF (10 ng/ml) and bFGF (10 ng/ml) for an additional 4 days. From day 6 onwards, the media was changed to IMDM/F12 supplemented with BMP4 (50 ng/ml), VEGF (10 ng/ml), EGF (10 ng/ml), TGF- $\alpha(20 \mathrm{ng} / \mathrm{ml})$, HGF (100 ng/ml), dexamethasone $\left(1 \times 10^{-7} \mathrm{M}\right.$; Sigma-Aldrich, St. Louis, MO), and DMSO (1\%, SigmaAldrich). From day 12 onwards, BMP4 and TGF- $\alpha$ were removed from the cocktail. For hepatocyte maturation, HGF, dexamethasone, and oncostatin $\mathrm{M}(20 \mathrm{ng} / \mathrm{ml})$ were included in the media from day 16 onwards. At day 19 post-differentiation, the iPSC-hepatocytes, or iHeps, were harvested for bioreactor culture. To compare the hepatic maturation status between bioreactor cultured iHeps and twodimensional mono-layer cultured iHeps, one plate of the cells was continuously differentiated until day 31 in parallel to bioreactor culture. At specific time points, markers for endoderm and hepatic lineage cells were assessed by immunocytochemistry (ICC), flow cytometry, and reverse transcription-quantitative PCR (RT-qPCR).

\section{Electron microscopic analysis}

One million hepatocytes (day 21 post-differentiation) were fixed with $4 \%$ glutaraldehyde at $4^{\circ} \mathrm{C}$ for overnight. Subsequently, the cells were dehydrated and embedded in resin for generation of thin sections for electron microscopic analysis. Harbor UCLA Electron Microscopy laboratory was used for sample preparation and image acquisition.

\section{Closed circuit hollow fiber bioreactor (HFB) system for culturing iHeps}

Preparation of HFB system: The closed-circuit bioreactor system consisting of a media reservoir, pumps, oxygenator, HFB cartridge containing cells, and flow path (tubing and adaptors). A polysulfone hollow fiber bioreactor cartridge (Alpha Plan, Germany) with a $70 \mathrm{~cm}^{2}$ inner surface area and a $0.21 \mu \mathrm{m}$ pore size was used for iHep culture. The hollow fibers are arranged in longitudinal fashion with a pack density of $36 \%-40 \%$ without waviness inside the durable polycarbonate casing of the cartridge. The HFB cartridge was connected with a reservoir bottle (FiberCell Systems Inc., USA) containing growth media, a membrane oxygenator (Radnoti, LLC., USA), and a pump (Masterflex L/S Digital Drive pump; Cole-Parmer, USA) by peroxide-cured silicon tubing (ColeParmer, USA). The media from the reservoir pumped into the HFB cartridge's vestibule is divided across the hollow fiber mouth for even flow into the ICS of fibers. Before entering into the cartridge, the media is oxygenated $\left(95 \% \mathrm{O}_{2}\right.$ and $\left.5 \% \mathrm{CO}_{2}\right)$ by an oxygenator module. This closedcircuit HFB system was placed in a cell culture incubator at $37^{\circ} \mathrm{C}$. An optimized protocol we developed for pre-culturing iHeps on microcarrier beads and subsequent culturing in HFB cartridges is given below.

Pre-culturing iHeps on microcarrier beads in spinner flask: A total of 9-10 million iHeps were harvested from the mono-layer culture plate and pre-cultured with laminin coated Cytodex microcarrier beads (Sigma-Aldrich) in HepatoZyme-SFM (Life Technologies) media supplemented with ITS (Insulin-Transferrin-Selenium, Life Technologies) and EGF $(25 \mathrm{ng} / \mathrm{ml})$ in a spinner culture bottle. The spinner bottle culture was performed in a $\mathrm{CO}_{2}$ incubator at $37^{\circ} \mathrm{C}$ with a stir speed of 40-70 RPM.

Loading iHeps into HFB system: Prior to cell loading, the bioreactor system was primed by perfusion with $500 \mathrm{ml}$ of PBS for 24 hours, then by $500 \mathrm{ml}$ of HepatoZyme-SFM media for 48 hours. For culturing cells, $250 \mathrm{ml}$ of HepatoZyme-SFM media supplemented with $2.5 \mathrm{ml}$ of ITS and EGF $(25 \mathrm{ng} / \mathrm{ml})$ was perfused. Before cell loading, the extracapillary space of the bioreactor was coated with the mouse laminin (Mouse Laminin I, Trevigen) according to the manufacturer's protocol. After 24 hours of pre-culturing in a spinner bottle, the cells on the microcarrier beads were harvested by centrifugation, resuspended in $3 \mathrm{ml}$ of HepatoZyme-SFM medium, and loaded into the extracapillary space of the HFB cartridge with a syringe. The media flow rate was set at $2 \mathrm{ml} / \mathrm{min}$. At indicated time points (day $0,3,6,9$, and 12) post-cell loading, $25 \mathrm{ml}$ of the culture medium was collected from the bioreactor media reservoir bottle and replaced with the same volume of freshly-made HepatoZyme-SFM medium containing ITS and EGF. The collected medium was stored at $-20^{\circ} \mathrm{C}$ for future assays. At day 12 post-loading, the cells in the ECS of the bioreactor were also harvested for RNA isolation.

\section{Reverse Transcription Quantitative PCR Analysis (RT-qPCR)}

Total RNA was extracted from iHeps cultured in plates or 
bioreactor using the RNeasy Mini Kit (QIAGEN) for RT-qPCR analysis [34]. Human primary hepatocyte RNA from fetal liver was included as a positive control (provided by Samuel W French, UCLA). RNA samples were reverse-transcribed using the SuperScript III Reverse Transcriptase kit (Life Technologies) with random primers as described by the manufacturers. Expression of human hepatic genes was quantified using the Platinum SYBR Green qPCR SuperMix-UDG with ROX Kit (Invitrogen) by the ViiA7 real-time PCR system (Applied Biosystems). Approximately $100 \mathrm{ng}$ of cDNA was used for each qPCR reaction. The reaction for each marker's gene expression was triplicated with the housekeeping human PPIG [peptidyl-prolyl isomerase G (cyclophilin $G)$ ] gene as an internal reference control. The sequences of the primer pairs used for qPCR are given in supplementary Table S1. All cDNAs were amplified under the following conditions: $50^{\circ} \mathrm{C}$ for $2 \mathrm{~min} ; 95^{\circ} \mathrm{C}$ for $2 \mathrm{~min}$ followed by 40 cycles of $95^{\circ} \mathrm{C}$ for $15 \mathrm{sec}$ and $60^{\circ} \mathrm{C}$ for $1 \mathrm{~min}$.

Human albumin, apolipoprotein B, prothrombin and glycogen assays

The iHep culture media samples from both the bioreactor and culture plate were collected. The amount of human albumin and apolipoprotein B produced by iHep was measured in triplicate by the Human Albumin ELISA kit (Bethyl Laboratories, Inc., Montgomery, TX) and the Human Apolipoprotein B ELISA kit (MABTECH, Inc) respectively. Prothrombin was measured by ELISA as per manufacturer's protocol (Molecular Innovations, Inc). Intra-cellular glycogen was detected by the Periodic Acid Schiff (PAS) assay as per manufacturer's protocol (Sigma-Aldrich).

\section{Flow Cytometry}

We followed a protocol described for flow cytometry analysis of stem cell derivatives [34]. The differentiating cells were collected at indicated time points, permeabilized using the Cytofix/Cytoperm kit (BD Biosciences), and analyzed using antibodies against SOX17 and CXCR4 (R \& D Systems), AFP (Dako, USA) Albumin (Bethyl Laboratories, Inc), and FOXA2 (Novus Biologicals, Littleton, CO). Data was acquired on a BD Fortessa (BD Biosciences) flow cytometer using FACSdiva software and analyzed using FlowJo software (TreeStar Inc.).

\section{Immunofluorescence assay}

Assay protocol has been described previously [35]. In brief, cells were fixed with methanol at day 4 post-differentiation. Following three PBS washes, the cells were blocked (10\% fetal bovine serum, $3 \%$ BSA, $0.1 \%$ Triton-x 100 in PBS) and incubated with human SOX17 mouse monoclonal primary antibodies (BD Biosciences) and FOXA2 rabbit monoclonal primary antibodies (Novus Biologicals, Littleton, $\mathrm{CO}$ ) at a dilution ratio of 1:200 for $5 \mathrm{hrs}$ to overnight at $4^{\circ} \mathrm{C}$. Either the goat anti-mouse polyclonal antibody (Alexa fluor 594) or goat anti-rabbit polyclonal antibody (Alexa fluor 488) was added as a secondary antibody (Life Technologies, USA) at 1:1000 dilutions and incubated for $1 \mathrm{hr}$ at room temperature. Between antibody changes the cells were washed thrice with PBS. The nuclei were stained with Hoechst dye (Life Technologies, USA). Stained cells were examined using a fluorescent microscope (Nikon Eclipse Ti-S, Japan).

\section{Statistical analysis}

Error bars reflect the standard deviation. P-values were determined by the two-tailed student's t-test and significance was reported as ${ }^{\star} \mathrm{p}<0.05 ;{ }^{* *} \mathrm{p}<0.01$ and ${ }^{* *} \mathrm{p}<0.001$.

\section{Results}

For the development of an iPSC-based bioartificial liver, the different components of the device including metabolically active iPSChepatocytes and the closed-circuit hollow fiber bioreactor system need to be integrated optimally. Figure 1 depicts various steps involved in iHep-BAL development. We utilized a human fibroblast-derived iPSC line for generating functional hepatocytes (Figure 2).

\section{Differentiation of human iPS cells into hepatocyte-like cells}

We adapted a three-stage differentiation program to differentiate iPSCs to mature hepatic lineage cells (Figure 2A). This process involves the first stage of definitive endoderm formation, a second stage of hepatic lineage specification, and the final third stage of hepatocyte maturation using various cocktails of cytokines and factors [34,36-38]. To accurately measure the differentiation efficiency on a single cell level, we employed flow cytometry to quantify cells that express endoderm and liver-specific markers. The cells were analyzed on day 4-5 for endoderm specific markers SOX17 and FOXA2 by immunocytochemistry (ICC) (Figure 2B), as well as by flow cytometry (SOX17 and CXCR4) (Figure 2C). SOX17 is a transcription factor specific for endoderm cells and CXCR4 is a chemokine receptor involved in cell migration during the gastrulation phase of embryonic development [39-41]. By day 15, we observed high levels of expression of alpha-fetoprotein (AFP), a marker for immature hepatic cells (Figure 2C). The liver-specific marker albumin was expressed by over $90 \%$ of the cells after day 15 , suggesting homogenous and efficient differentiation. By day 21 as the hepatic cells proceed to a more mature phenotype, we observed reduced expression of AFP. Below we describe the functional evaluation of differentiated iPSC-hepatocytes.

\section{Functional analysis of iPSC-hepatocytes}

Generating functional hepatocytes from iPSCs is critical for the successful development of an iHep-based bioartificial liver device that can substitute for the metabolic and detoxification functions of the liver. Therefore, we assessed the activities of cytochrome p450

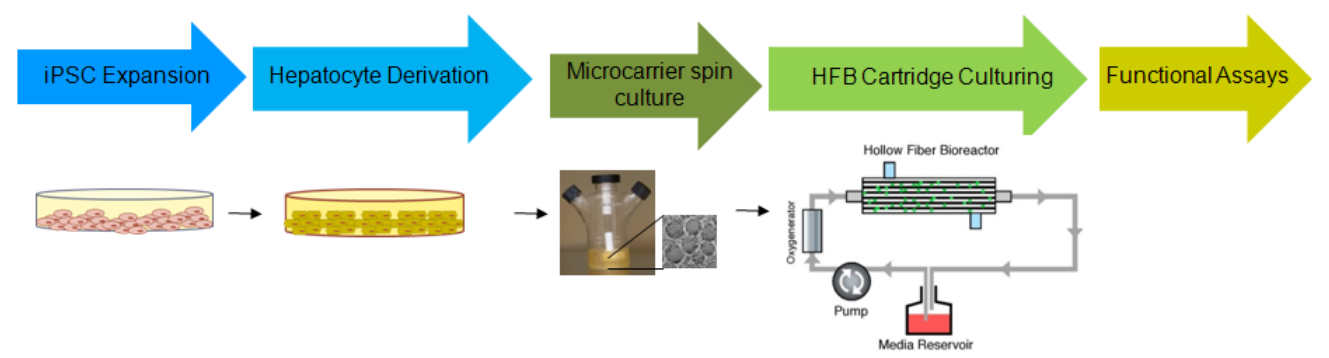

Figure 1: A schematic illustration of various steps involved in iPSC-hepatocytes based bioartificial liver device. The iPSCs are expanded and differentiated to hepatocytes. The iPSC-derived hepatocytes are loaded in the bioreactor for pre-clinical functional evaluation. 
A.

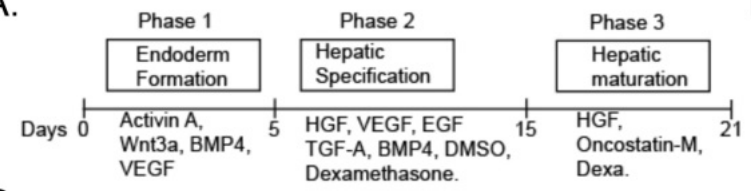

C.
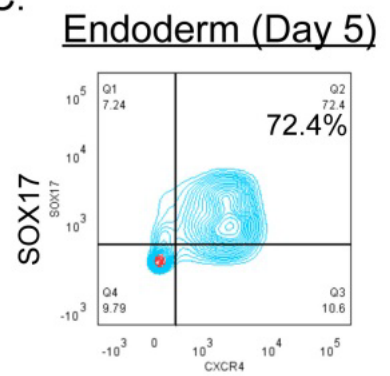

CXCR4

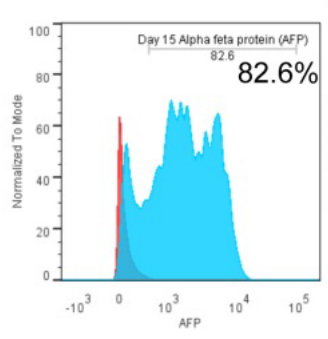

AFP
B.

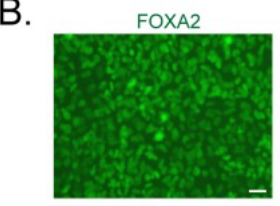

toblast (Day 15)

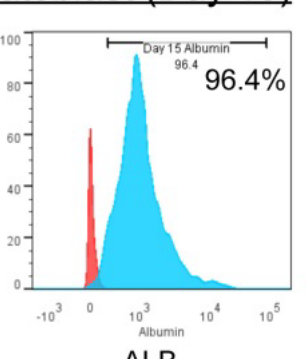

ALB
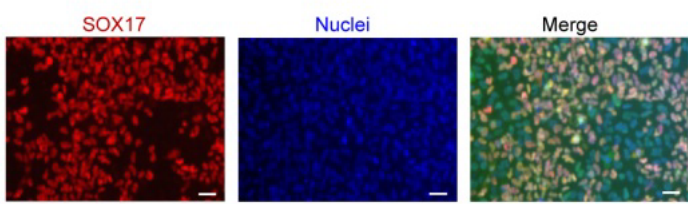

iPSC-Hepatocyte (Day 21)
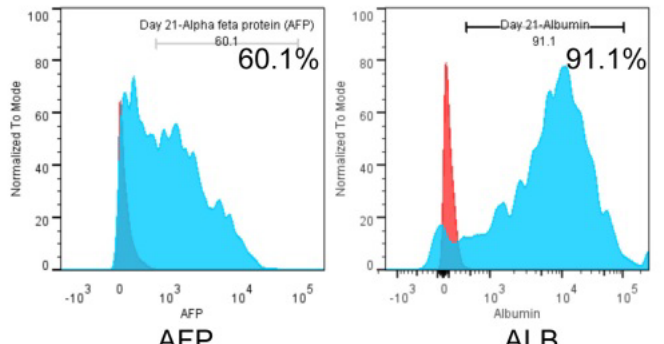

\section{Phase I Enzymes}
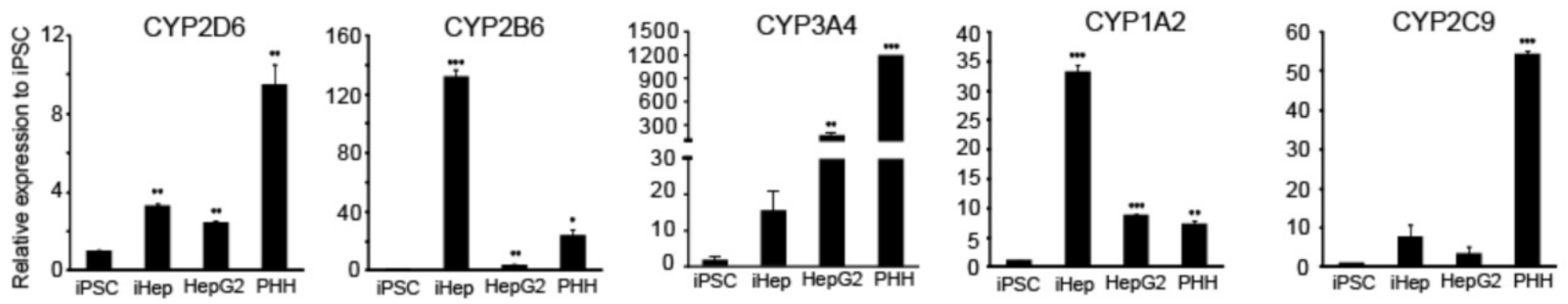

\section{E. Phase II Enzymes}
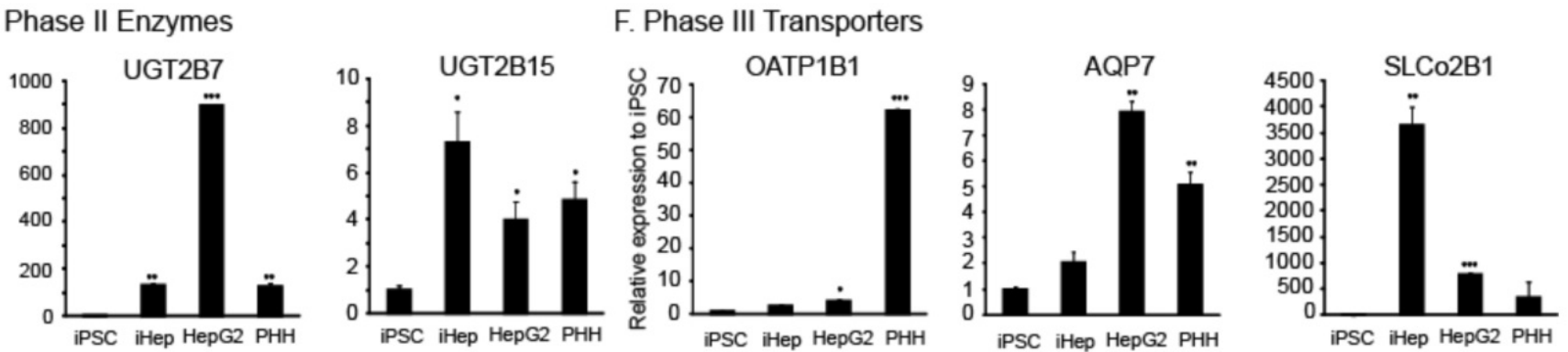

Figure 2: Differentiation of iPSCs into hepatic lineage cells. (A) Schematic showing the experimental outline of differentiation of human iPSC into functional hepatocytes (B) Immunocytochemistry of endoderm marker expression at day 4 differentiation (scale bar $25 \mu \mathrm{m}$ ). (C) Flow cytometry analysis of endoderm marker expression at day 5 differentiation. AFP and albumin (ALB) producing cell population at day 15 and day 21 of differentiation. (D, E and F) Phase I, II, and III CYP gene expression analysis. Relative gene expression to undifferentiated iPSCs was calculated for iHeps, HepG2, and primary human hepatocytes (PHH) and presented in the bar graphs with standard deviations. Representative data from three independent experiments are presented.

(CYP) and carbohydrate metabolic pathways in the iHeps. The liver CYP system is crucial for degradation and clearance of endogenous metabolites, hormones and xenobiotics [42-48]. CYP genes are classified as Phase I and Phase II enzymes and Phase III transporters. Phase I enzymes function in oxidation-reduction reactions and Phase II enzymes act in modifying metabolites by acetylation, sulfation, glucuronidation and glutathione conjugation. Phase III transporters are involved in drug clearance. CYP components have been investigated extensively for their roles in metabolism and excretion of pharmaceutical compounds [42-46,49,50]. We have observed that the basal level expression of many CYP genes, including CYP2D6, CYP2B6, CYP1A2, UGT2B7, UGT2B15, and SLCo2B1 were significantly upregulated in iPSC-hepatocytes compared to that of undifferentiated iPSCs (Figures 2D-2F). The basal level of CYP3A4 expression was increased in iHep, but was not significant. We have also observed that CYP genes were differentially expressed in iHeps, fetal hepatocytes, and HepG2. CYP genes are induced upon exposure of hepatocytes to various xenobiotics and drugs. We used the drug rifampicin, which is metabolized by liver, to study CYP activity. The iPSC-hepatocytes (20 days post-differentiation) were treated with rifampicin $(10 \mu \mathrm{M})$ for 48 hours, and the CYP3A gene expression was quantified. Though iHep had a low basal level of CYP3A4, it was induced upon treatment with the rifampicin (Figure $3 \mathrm{~A}$ ), indicating the metabolic maturity of iHeps.

Glycogen, a branched polysaccharide, is stored in liver cells. To test the capacity of the glycogen storage by the iHeps, we performed a Periodic Acid Schiff (PAS) assay. The differentiated iPSC-hepatocytes (day 20 post-differentiation) were fixed and stained for glycogen. The cells that reacted with the PAS stain were observed with microscope 


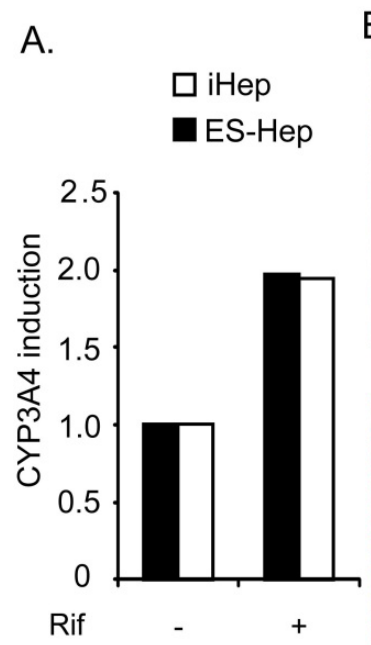

B.

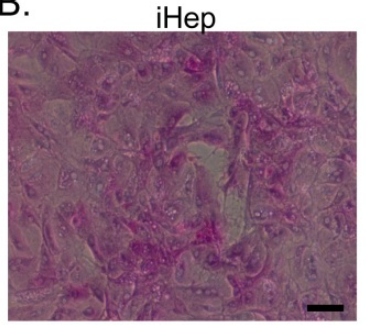

Huh-7.5.1

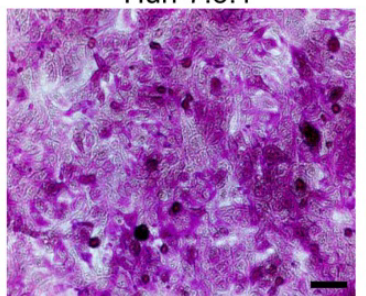

C.

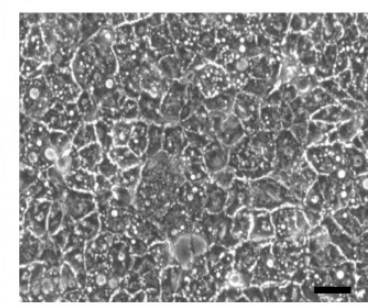

D.

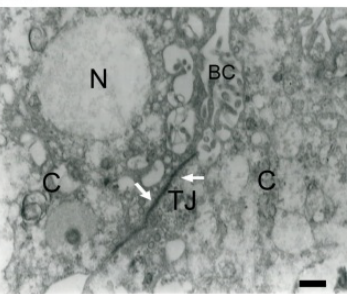

Figure 3: Functional and morphological analysis of hepatic cells derived from iPSC. (A) Induction of CYP34A mRNA expression by rifampicin stimulation on day 20 hepatic lineage cells derived from H9 ES and iPS cells. Housekeeping gene (PPIG) normalized CYP3A4 mRNA level of unstimulated cells was used for calculating fold induction. (B) PAS staining for glycogen storage in iPSC-derived hepatocytes (scale bar $10 \mu \mathrm{m}$ ). Liver cancer cell line Huh-7.5.1 is included as a control. (C) Bright field microscopic image shows the polygonal morphology of iPSC-derived hepatocytes with defined tight junctions at day 21 post-differentiation (scale bar $10 \mu \mathrm{m}$ ). (D) Ultra-structural analysis of iPSC-derived hepatocytes (day 15) reveals formation of hepatic features such as tight junctions (TJ; arrows) and biliary canaliculi (BC) between two adjacent cells (scale bar $0.25 \mu \mathrm{m}$ ). The cells were grown as a two-dimensional monolayer culture before processing for an electron microscopy study. (N: nucleus; C: cytoplasm).

A.

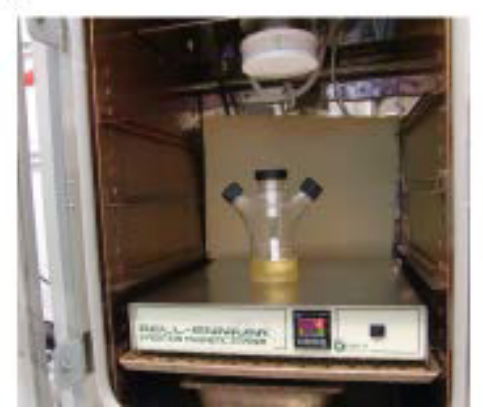

B.

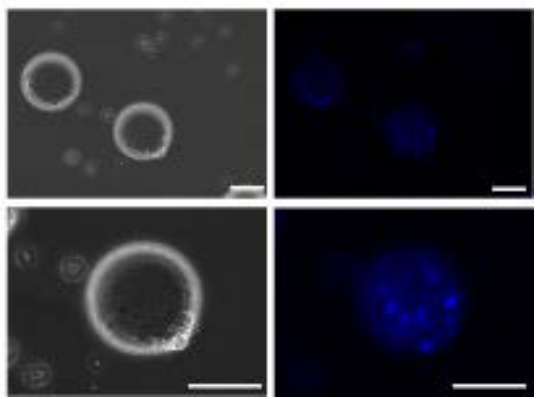

Figure 4: Suspension culture of iHeps on dextran microcarrier beads. (A) The iPSC-hepatocytes culture in a spinner flask is shown in the context of a cell culture incubator. (B) Bright field and fluorescent images show hepatocytes attached onto dextran microcarrier spheres (scale bar $50 \mu \mathrm{m}$ ). For nuclear staining, cells containing beads were incubated with Hoechst dye for 10 minutes.

and images were obtained. Hepatocytes derived from 83iCTR show glycogen staining, as do the positive control Huh-7.5.1 hepatoma cell line (Figure 3B). The differentiated iPSC-hepatocytes exhibited polygonal morphology with tight junctions in a monolayer culture condition (Figure 3C). Ultra-structural analysis demonstrated formation of putative bile canaliculi by adjacent hepatic cells (Figure 3D). Taken together, our results showed that the iPSC-differentiated hepatocytes exhibit the hepatic cell phenotype and are metabolically active. Next, we focused on utilizing the iHeps for the development of a bioartificial liver.

\section{Design, process development and in vitro functional study of bioartificial liver}

The hollow fiber bioreactor cartridge loaded with functional hepatocytes on the extra capillary space would serve as bioartificial liver module. Below, we have provided the experimental steps involved in BAL development and the results of a functional study.
Large-scale culturing of iPSC-hepatocytes on microcarriers using a spinner flask bioreactor: Large-scale production of iPSCs and iPSC-hepatocytes is a key step in the development of an iHep based BAL. For clinical application, estimated 5-20 billion hepatocytes are required for the treatment of patient with decompensated liver disease. To achieve this, microcarriers are useful for cultivating anchorage-dependent cells in suspension, which allows large-scale and high-yield production of iHeps. For a proof-of-concept study, we used dextran microcarrier beads $(60-87 \mu \mathrm{m}$ in size) for culturing iHeps. The microcarriers were coated with laminin and 10 million iHeps (19 day post-differentiation) were added to the microcarrier in a $250 \mathrm{ml}$-spinner flask (Figure 4A). Cells were maintained in hepatocyte-differentiation media for $24 \mathrm{hrs}$ as a suspension culture in a $\mathrm{CO}_{2}$ incubator at $37^{\circ} \mathrm{C}$. Culture samples were collected for microscopic examination. We observed that the iPSC-hepatocytes were attached to the surface of microcarrier spheres (Figure $4 \mathrm{~B}$ ). A trypan blue dye exclusion test indicated that the attached cells were viable. Subsequently, the iHep-beads were transferred to continuous- 
Citation: Ren S, Ignatius Irudayam J, Contreras D, Sareen D, Talavera-Adame D, et al. (2015) Bioartificial Liver Device Based on Induced Pluripotent Stem Cell-Derived Hepatocytes. J Stem Cell Res Ther 5: 263. doi:10.4172/2157-7633.1000263

Page 6 of 9

flow hollow fiber bioreactor system.

Hollow fiber bioreactor system for culturing and functional assessment of the iHep-BAL: In order to maintain physiological functions, the iPSC-hepatocytes loaded in the bioartificial liver device have to survive in the extra-capillary space microenvironment over a period of time and exchange gas, nutrients, proteins, and metabolites. These in vitro parameters of cell viability and functions can be used for assessing the quality and functionality of the iHep-BAL. The iHeps $\left(1 \times 10^{7}\right.$ cells $)$ attached to the microcarrier beads were loaded into the extracapillary space of a polysulfone hollow fiber bioreactor cartridge and the oxygenated culture media was perfused continuously for a total of 12 days (Figure 5). Media samples were collected from ICS flowthrough at different time points (days 3,6,9 and 12) for functional assays. First, we set out to characterize cell viability and growth. Harvesting cells from the ECS of the hollow fiber bioreactor proved to be an inefficient process ( $20 \%$ yield of total input cells) due to the cells on beads getting stuck in between the narrow spaces of hollow micro fibers. However, the harvested cells at day 12 had over $80 \%$ viability. Subsequently, we utilized the iHep functional parameters described below as indicators for cell health.

Secretion of plasma proteins by iHeps: Liver-specific proteins and metabolites produced by the iHeps were secreted into ICS through $0.21 \mu \mathrm{m}$ pores on the hollow fibers. This design is similar to in vivo natural liver architecture where the hepatocytes secrete plasma proteins into liver sinusoidal spaces. For functional verification, human plasma proteins albumin, apoB and prothrombin secreted by iHeps into the ICS media were quantified (Figures 6A-6C). We observed a significant
A.

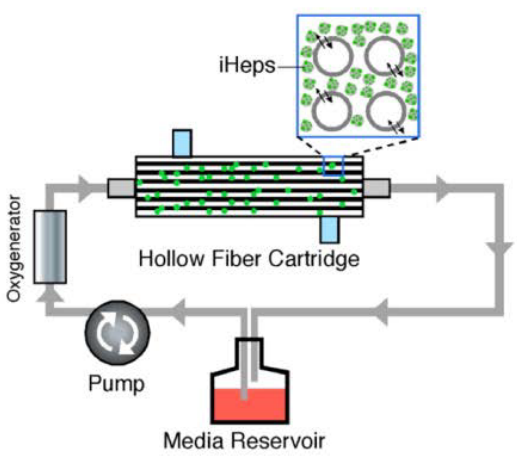

B.

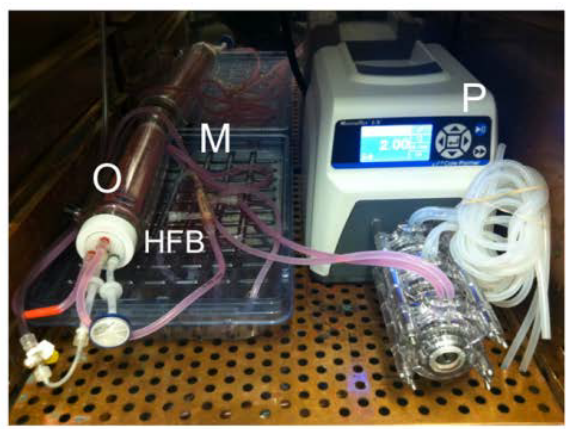

Figure 5: Closed-circuit hollow fiber bioreactor system for culturing iPSC-hepatocytes. (A) Schematic diagram displaying the various components of HFB culture setup. The membranous hollow fibers are encased in a cartridge and each fiber is connected to vestibules at the inflow and outflow ends. The functional cells adhere to the outside of each fiber. The hollow fiber contains numerous micropores that allow the exchange of metabolites and proteins. The cells are bathed in a continuous flow of oxygenated growth media pumped from a media reservoir. (B) The closed-circuit continuous flow setup including hollow fiber bioreactor (HFB) cartridge loaded with iPSC-hepatocytes is shown inside a $37^{\circ} \mathrm{C}$ incubator. The iPSC-hepatocyte loaded HFB cartridge would function as a bioartificial liver device. P: pump; O: membrane oxygenator; M: media bag inside the tray.
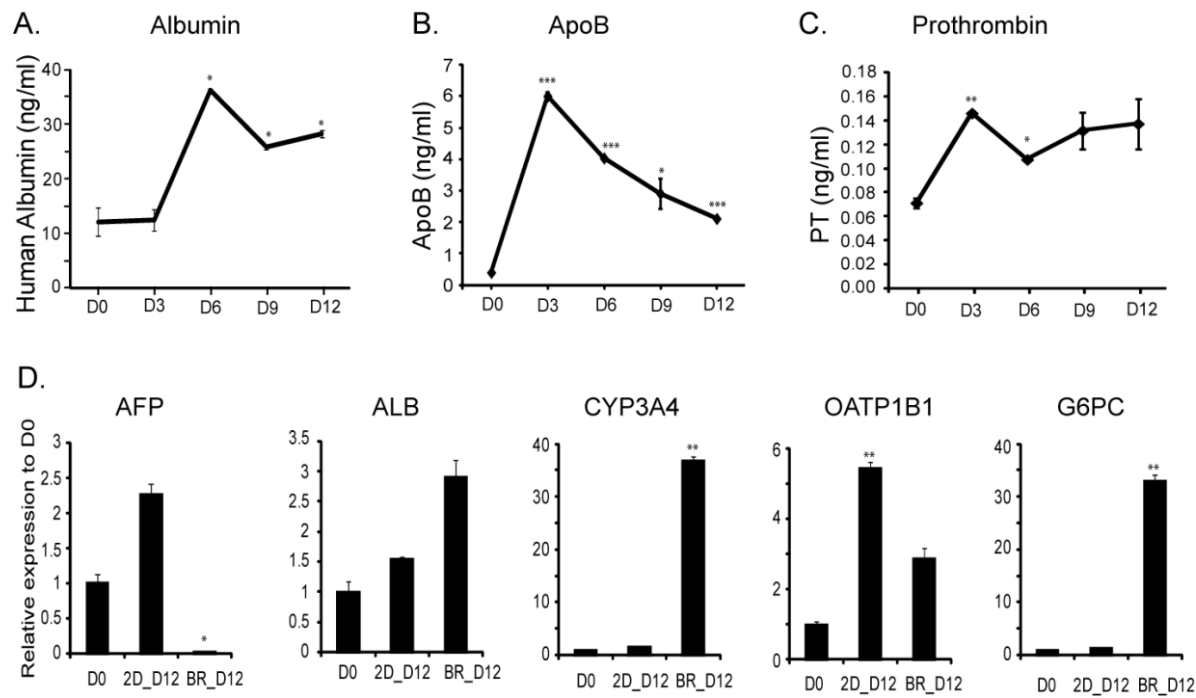

Figure 6: Functional assessment of iHeps cultured in the hollow fiber bioreactor cartridge with continuous flow. (A, B, C) Plasma proteins albumin, apolipoprotein B, and prothrombin secretion by iHeps at indicated time points are shown. The mean values with standard deviations are presented in the graphs. (D) Relative gene expression to input day 0 iPSC-hepatocytes was calculated and values are shown in the graphs with standard deviations. Note the significant reduction of AFP level in cells cultured on a continuous flow condition. 2D: two-dimensional culturing in dish; BR: bioreactor culturing. Three independent experiments were performed and representative data is shown. 
increase in human albumin secretion after day 3 of iHep culturing in the cartridge. ApoB and prothrombin levels were increasing form D0 to D3 and decreased thereafter. Liver enzyme ALT was below detectable level in all the time points indicating better cell survival (data not shown).

Improved maturation of iHeps by continuous media flow: In vivo, the generated metabolites and toxic waste products by hepatocytes are continuously removed by the blood flow resulting in maintenance of liver homeostasis. The closed-circuit flow system can mimic the in vivo physiological condition more effectively compared to that of cells cultured in a two-dimensional static condition. Thus, we further characterized the iHeps cultured in the HF bioreactors. At the end of the experiment on day 12 , cells on beads were harvested from the bioreactors for gene expression analysis. Culturing iPSC-hepatocytes in a continuous flow micro-environment on laminin coated beads improved the maturation state, as evidenced by 83 -fold reduction of AFP and significant increase in CYP3A4 and Glucose-6-phosphatase (G6PC) gene expression compared to that of input Day 0 iHeps (Figure 6D). ALB and OATP1B1 expression was not significantly altered in the continuous flow condition. The hepatic markers CYP3A4 and G6PC were also upregulated in continuous flow cultured iHeps compared to that of parallel static 2D plate cultured iHeps. In conclusion, our results indicated that the iPSC-hepatocytes cultured in the HFB maintained the plasma protein secretory function with an improved hepatic maturation signature.

\section{Discussion}

Here we present a proof-of-concept preclinical in vitro study on the development of a prototype iPSC-hepatocyte-based bioartificial liver module. The biological factors and chemicals required for deriving functional hepatocytes from pluripotent stem cells have been studied extensively [34,36-38,51-62]. Using the established three-phase differentiation protocol, we have generated an over $90 \%$ homogenous population of hepatic lineage cells (Figure 2). The iPSC-hepatocytes had active cytochrome p450 and functional lipid and carbohydrate metabolic pathways (Figures 2 and 3). We observed that cytochrome p450 genes and transporters were differentially expressed in iHeps, fetal hepatocytes and HepG2. This is not surprising given the variation in CYP gene induction kinetics and CYP polymorphisms (pharmacogenetics) in individuals of different genetic backgrounds that have been shown to affect drug metabolism and clearance $[45,46,63,64]$. Establishing a repertoire of well-characterized iPSC lines from individuals of diverse genetic backgrounds and/or collecting iPSC lines with genetically engineered CYP genes can be useful for the treatment of the wide range of liver diseases due to drug overdoses or altered CYP metabolic activity.

In general, hepatic cells derived from pluripotent stem cells using current differentiation protocols are immature, as demonstrated by the presence of alpha-fetoprotein (Figure 2C). In adult livers, AFP expression is below detectable levels. Interestingly, we observed that the iHeps on laminin coated beads cultured in the hollow fiber cartridge with continuous flow showed about 83-fold reduction in AFP expression with concomitant increase in mature liver markers (Figure 6). This finding has great practical value, as mature hepatocytes are critical for assessing the toxicity and pharmacokinetic properties of drug compounds during the pre-clinical development phase.

For prototype iHep-BAL development, we have chosen a hollow fiber capillary design because of its high surface area and resemblance to natural blood capillary liver microarchitecture for nutrient and gas exchange. This design has been used in other liver support devices such as the extracorporeal liver assist device (ELAD), and HepatAssist liver support system $[11,26]$. Additional design features include the cartridge having layers of polysulfone membrane sheets where alternate layers filled with cells or blood/plasma flow can be considered. Another design consideration is improving the throughput. In this study, we have used a single cartridge closed-circuit system. For testing multiple growth conditions, animal experiments, and human clinical studies, a multi-cartridge system can be useful. An integrated multi-cartridge system can allow for parallel production, better quality control, and uniformity among bioartificial liver devices.

We have utilized dextran microcarrier beads for growing iHeps in suspension culture (Figure 4). An additional advantage of using microcarrier suspension, besides for a large-scale culturing purpose, is that iHep-beads can be easily transferred between culture modules without having the stress of an enzymatic dissociation process such as trypsinization. The iHep-microcarrier culture approach can also provide additional benefits such as minimizing cells clogging the pores in the hollow fiber. If the cells are directly attaching and expanding on the surface of hollow fiber, there is a high possibility of pore obstruction, which can prevent the exchange of gas, metabolites, and nutrients in and out of the fiber capillaries.

In this study, we mainly focused on developing various steps in iHep-BAL set up and validating iHep functions by in vitro studies. This prototype iHep-BAL can be further developed and validated for rescuing disease-specific decompensated liver functions. In acute liver failure due to acetaminophen (APAP; paracetamol) poisoning, the liver cannot detoxify ammonia and secrete blood coagulation proteins such as prothrombin, resulting in increased intracranial pressure and bleeding $[65,66]$. Prothrombin produced by iHep in BAL can mitigate this bleeding issue. APAP metabolism and lethal concentration, as well as ammonia detoxification production by iHeps needed to be assessed.

For clinical applications, the iHep-BAL device has to reconstitute

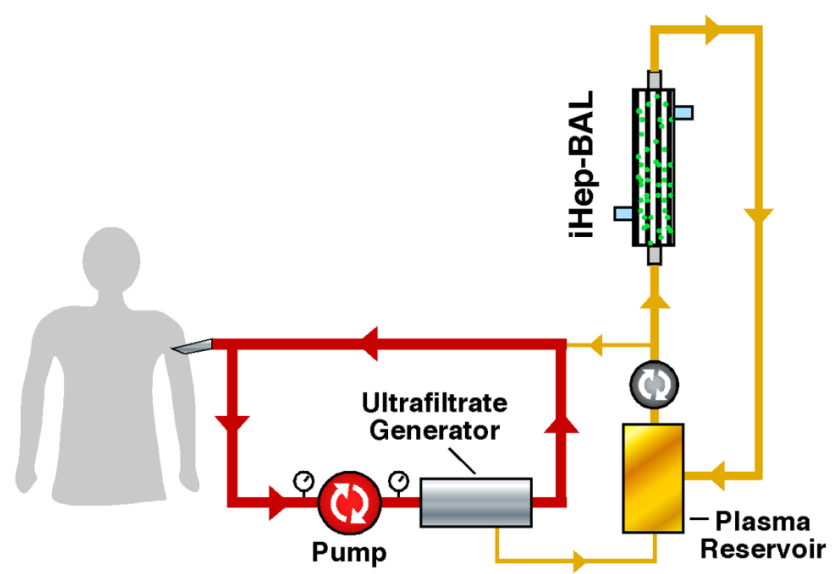

Figure 7: Outline of iHep-based extra-corporeal closed circuit BAL system for supporting patients with liver failure. Blood drawn from patient through a duallumen catheter is pumped through ultrafilterate generator to separate plasma from the cellular components. The arrows indicate the direction of the flow. The plasma (orange line) is then passed through a hollow fiber cartridge containing iHeps that can remove toxic metabolites. The detoxified plasma is routed through a plasma reservoir for subsequent rounds of detoxification. Part of the detoxified plasma is diverted back to blood cell component path and will enter the body-circulation through the dual-lumen catheter. This extra-corporeal closed circuit BAL system can restore some of the liver's vital functions and allow time for the patient's liver to repair and regenerate, thus avoiding a transplant, or act as bridge to transplantation. 
Citation: Ren S, Ignatius Irudayam J, Contreras D, Sareen D, Talavera-Adame D, et al. (2015) Bioartificial Liver Device Based on Induced Pluripotent Stem Cell-Derived Hepatocytes. J Stem Cell Res Ther 5: 263. doi:10.4172/2157-7633.1000263

the function of the damaged liver and improve the health condition of the patient. In vivo preclinical studies in rat and pig model systems of liver failure will provide insights into the safety and efficacy of an iHepBAL device. For preclinical testing, acute liver failure animal models including anhepatic rats and acetaminophen toxicity in pigs can be used $[67,68]$. Based on plasma proteins, albumin, and apoB secretion data (Figure 6), the iHeps can be cultured for 2 days in the cartridge after loading and subsequently be used for liver support purposes for in vivo testing. We have utilized a $70 \mathrm{~cm}^{2}$ mini-cartridge for this proof-of-concept study and testing the efficacy of the iHep-BAL in a large animal setting requires larger HFB cartridges $\left(6000 \mathrm{~cm}^{2}\right)$ with a loading density of over $1 \times 10^{9}$ cells. The scaling up of iHep production is a key component, which can be accomplished by differentiating and expanding cells on microcarriers in a suspension culture. Figure 7 shows a concept outline of iHep-based extra-corporeal closedcircuit BAL system for supporting liver failure patients, where the patient's plasma will be circulated through the iHep loaded cartridge for detoxification and plasma protein reconstitution. In conclusion, we have established a prototype iHep-BAL that can be further developed as a liver assist device for the treatment of patients with acute liver failure or decompensated chronic hepatitis.

\section{Acknowledgment}

We thank Seigo Hatada (Cedars-Sinai Medical Center, USA) for graphics and illustrations. We thank Nicholas Ten (Yale University, USA) for language editing and formatting the manuscript. The Huh-7.5.1 cell line was a kind gift from Francis V. Chisari (The Scripps Research Institute, USA). We thank Samuel W. French (Harbor-UCLA Medical Center, USA) for assistance with the electron microscopy study. This work was funded by Cedars-Sinai Medical Center's Institutional Research Award to V.A.

\section{References}

1. CDC (2014) Centers for Disease Control and Prevention. Hepatitis Awareness.

2. Hoofnagle JH, Carithers RL Jr., Shapiro C, Ascher N (1995) Fulminant hepatic failure: summary of a workshop. Hepatology 21: 240-252. [PubMed]

3. Bernal W, Auzinger G, Dhawan A, Wendon J (2010) Acute liver failure. Lancet 376: 190-201. [PubMed]

4. Carpentier B, Gautier A, Legallais C (2009) Artificial and bioartificial liver devices: present and future. Gut 58: 1690-1702. [PubMed]

5. Ellis AJ, Hughes RD, Wendon JA, Dunne J, Langley PG, et al. (1996) Pilotcontrolled trial of the extracorporeal liver assist device in acute liver failure. Hepatology 24: 1446-1451. [PubMed]

6. MacDonald JM, Wolfe SP, Roy-Chowdhury I, Kubota H, Reid LM (2001) Effect of flow configuration and membrane characteristics on membrane fouling in a novel multicoaxial hollow-fiber bioartificial liver. Ann N Y Acad Sci 944: 334-343. [PubMed]

7. Rocen M, Kieslichova E, Merta D, Uchytilova E, Pavlova Y, et al. (2010) The effect of Prometheus device on laboratory markers of inflammation and tissue regeneration in acute liver failure management. Transplant Proc 42: 3606-3611. [PubMed]

8. Sussman NL, Chong MG, Koussayer T, He DE, Shang TA, et al. (1992) Reversal of fulminant hepatic failure using an extracorporeal liver assist device. Hepatology 16: 60-65. [PubMed]

9. Iwata $\mathrm{H}$, Ueda $\mathrm{Y}$ (2004) Pharmacokinetic considerations in development of a bioartificial liver. Clin Pharmacokinet 43: 211-225. [PubMed]

10. Demetriou AA (2005) Hepatic assist devices. Panminerva Med 47: 31-37. [PubMed]

11. Demetriou AA, Brown RS Jr., Busuttil RW, Fair J, McGuire BM, et al. (2004) Prospective, randomized, multicenter, controlled trial of a bioartificial liver in treating acute liver failure. Ann Surg 239: 660-667. [PubMed]

12. Rozga J, Williams F, Ro MS, Neuzil DF, Giorgio TD, et al. (1993) Development of a bioartificial liver: properties and function of a hollow-fiber module inoculated with liver cells. Hepatology 17: 258-265. [PubMed]
13. Kiley JE, Welch HF, Pender JC, Welch CS (1956) Removal of blood ammonia by hemodialysis. Proc Soc Exp Biol Med 91: 489-490. [PubMed]

14. Parbhoo SP, James IM, Ajdukiewicz A, Xanalatos C, Kennedy J, et al. (1971) Extracorporeal pig-liver perfusoon in treatment of hepatic coma due to fulminant hepatitis. Lancet 1: 659-665. [PubMed]

15. Sugiyama N, Rozga J, Demetriou AA (2001) Biological extracorporeal liver assist devices: A minireview. Transplant Rev 15: 129-141.

16. Hui T, Rozga J, Demetriou AA (2001) Bioartificial liver support. J Hepatobiliary Pancreat Surg 8: 1-15. [PubMed]

17. Watanabe FD, Mullon CJ, Hewitt WR, Arkadopoulos N, Kahaku E et al. (1997) Clinical experience with a bioartificial liver in the treatment of severe liver failure. A phase I clinical trial. Ann Surg 225: 484-491. [PubMed]

18. Laleman W, Wilmer A, Evenepoel P, Elst IV, Zeegers M, et al. (2006) Effect of the molecular adsorbent recirculating system and Prometheus devices on systemic haemodynamics and vasoactive agents in patients with acute-onchronic alcoholic liver failure. Crit care 10: R108. [PubMed]

19. Ichai P, Samuel D (2004) Treatment of patients with hepatic failure: the difficult place of liver support systems. J Hepatol 41: 694-695. [PubMed]

20. Rifai K, Ernst T, Kretschmer U, Bahr MJ, Schneider A, et al. (2003) Prometheus-a new extracorporeal system for the treatment of liver failure. J Hepatol 39: 984-990. [PubMed]

21. Matsubara S, Okabe K, Ouchi K, Miyazaki Y, Yajima Y, et al. (1990) Continuous removal of middle molecules by hemofiltration in patients with acute liver failure. Crit Care Med 18: 1331-1338. [PubMed]

22. O'Grady JG, Gimson AE, O'Brien CJ, Pucknell A, Hughes RD, et al. (1988) Controlled trials of charcoal hemoperfusion and prognostic factors in fulminant hepatic failure. Gastroenterology 94: 1186-1192. [PubMed]

23. Nyberg SL, Remmel RP, Mann HJ, Peshwa MV, Hu WS, et al. (1994) Primary hepatocytes outperform Hep G2 cells as the source of biotransformation functions in a bioartificial liver. Ann Surg 220: 59-67. [PubMed]

24. Hughes RD, Nicolaou N, Langley PG, Ellis AJ, Wendon JA, et al. (1998) Plasma cytokine levels and coagulation and complement activation during use of the extracorporeal liver assist device in acute liver failure. Artif Organs 22 : 854-858. [PubMed]

25. Moussy Y (2003) Convective flow through a hollow fiber bioartificial liver. Artif Organs 27: 1041-1049. [PubMed]

26. Sussman NL, Kelly JH (1993) Improved liver function following treatment with an extracorporeal liver assist device. Artif Organs 17: 27-30. [PubMed]

27. Rozga J, Podesta L, LePage E, Morsiani E, Moscioni AD, et al. (1994) A bioartificial liver to treat severe acute liver failure. Ann Surg 219: 538-544. [PubMed]

28. Wang L, Sun J, Li L, Mears D, Horvat M, et al. (1998) Comparison of porcine hepatocytes with human hepatoma (C3A) cells for use in a bioartificial liver support system. Cell Transplant 7: 459-468. [PubMed]

29. Patience C, Takeuchi Y, Weiss RA (1997) Infection of human cells by an endogenous retrovirus of pigs. Nat Med 3: 282-286. [PubMed]

30. Roberts EA, Letarte M, Squire J, Yang S (1994) Characterization of human hepatocyte lines derived from normal liver tissue. Hepatology 19: 1390-1399. [PubMed]

31. Al-Nedawi K, Meehan B, Micallef J, Lhotak V, May L, et al. (2008) Intercellular transfer of the oncogenic receptor EGFRvIII by microvesicles derived from tumour cells. Nat Cell Biol 10: 619-624. [PubMed]

32. Rak J (2010) Microparticles in cancer. Semin Thromb Hemost 36: 888-906. [PubMed]

33. Sareen D, Ebert AD, Heins BM, McGivern JV, Ornelas L, et al. (2012) Inhibition of apoptosis blocks human motor neuron cell death in a stem cell model of spinal muscular atrophy. PloS one 7: e39113. [PubMed]

34. Cheng X, Ying L, Lu L, Galvao AM, Mills JA, et al. (2012) Self-renewing endodermal progenitor lines generated from human pluripotent stem cells. Cell stem cell 10: 371-384. [PubMed]

35. Chu D, Ren S, Hu S, Wang WG, Subramanian A, et al. (2013) Systematic analysis of enhancer and critical cis-acting RNA elements in the proteinencoding region of the hepatitis C virus genome. J Virol 87: 5678-5696. 
Citation: Ren S, Ignatius Irudayam J, Contreras D, Sareen D, Talavera-Adame D, et al. (2015) Bioartificial Liver Device Based on Induced Pluripotent Stem Cell-Derived Hepatocytes. J Stem Cell Res Ther 5: 263. doi:10.4172/2157-7633.1000263

\section{[PubMed]}

36. Rashid ST, Corbineau S, Hannan NJ, Marciniak SJ, Miranda E, et al. (2010) Modeling inherited metabolic disorders of the liver using human induced pluripotent stem cells. J Clin Invest 120: 3127-3136. [PubMed]

37. Basma H, Soto-Gutierrez A, Yannam GR, Liu L, Ito R, et al. (2009) Differentiation and transplantation of human embryonic stem cell-derived hepatocytes. Gastroenterology 136: 990-999. [PubMed]

38. Chen YF, Tseng CY, Wang HW, Kuo HC, Yang VW, et al. (2012) Rapid generation of mature hepatocyte-like cells from human induced pluripotent stem cells by an efficient three-step protocol. Hepatology 55: 1193-1203. [PubMed]

39. McGrath KE, Koniski AD, Maltby KM, McGann JK, Palis J (1999) Embryonic expression and function of the chemokine SDF-1 and its receptor, CXCR4. Dev Biol 213: 442-456. [PubMed]

40. Nair S, Schilling TF (2008) Chemokine signaling controls endodermal migration during zebrafish gastrulation. Science 322: 89-92. [PubMed]

41. Tam PP, Kanai-Azuma M, Kanai Y (2003) Early endoderm development in vertebrates: lineage differentiation and morphogenetic function. Curr Opin Genet Dev 13: 393-400. [PubMed]

42. Parker AJ, Houston JB (2008) Rate-limiting steps in hepatic drug clearance: comparison of hepatocellular uptake and metabolism with microsomal metabolism of saquinavir, nelfinavir, and ritonavir. Drug Metab Dispos 36: 13751384. [PubMed]

43. Lau YY, Sapidou E, Cui X, White RE, Cheng KC (2002) Development of a novel in vitro model to predict hepatic clearance using fresh, cryopreserved, and sandwich-cultured hepatocytes. Drug Metab Dispos 30: 1446-1454. [PubMed]

44. McLean AE (1967) Effect of hexane and carbon tetrachloride on microsomal cytochrome (P450). Biochem Pharmacol 16: 2030-2033. [PubMed]

45. Iyer L, Ratain MJ (1998) Pharmacogenetics and cancer chemotherapy. Eur J Cancer 34: 1493-1499. [PubMed]

46. Guengerich FP (2004) Cytochrome P450: what have we learned and what are the future issues? Drug Metab Rev 36: 159-197. [PubMed]

47. Monostory K, Dvorak Z (2011) Steroid regulation of drug-metabolizing cytochromes P450. Curr Drug Metab 12: 154-172. [PubMed]

48. Zimniak P, Waxman DJ (1993) Liver Cytochrome P450 Metabolism of Endogenous Steroid Hormones, Bile Acids, and Fatty Acids. Springer, Berlin, Heidelberg.

49. Sengupta S, Johnson BP, Swanson SA, Stewart R, Bradfield CA, et al. (2014) Aggregate Culture of Human Embryonic Stem Cell-Derived Hepatocytes in Suspension is an Improved in Vitro Model for Drug Metabolism and Toxicity Testing. Toxicol Sci 140: 236-245. [PubMed]

50. Du Y, Wang J, Jia J, Song N, Xiang C, et al. (2014) Human hepatocytes with drug metabolic function induced from fibroblasts by lineage reprogramming. Cell stem cell 14: 394-403. [PubMed]

51. Lavon N, Yanuka O, Benvenisty N (2004) Differentiation and isolation of hepatic-like cells from human embryonic stem cells. Differentiation 72: 230 238. [PubMed]

52. Ruhnke M, Ungefroren H, Nussler A, Martin F, Brulport M, et al. (2005) Differentiation of in vitro-modified human peripheral blood monocytes into hepatocyte-like and pancreatic islet-like cells. Gastroenterology 128: 1774 1786. [PubMed]

53. Hay DC, Fletcher J, Payne C, Terrace JD, Gallagher RC, et al. (2008) Highly efficient differentiation of hESCs to functional hepatic endoderm requires ActivinA and Wnt3a signaling. Proc Natl Acad Sci USA 105: 12301-12306. [PubMed]

54. Hay DC, Zhao D, Fletcher J, Hewitt ZA, McLean D, et al. (2008) Efficient differentiation of hepatocytes from human embryonic stem cells exhibiting markers recapitulating liver development in vivo. Stem cells 26: 894-902. [PubMed]

55. Campard D, Lysy PA, Najimi M, Sokal EM (2008) Native umbilical cord matrix stem cells express hepatic markers and differentiate into hepatocyte-like cells. Gastroenterology 134: 833-848. [PubMed]

56. Cai J, Zhao Y, Liu Y, Ye F, Song Z, et al. (2007) Directed differentiation of human embryonic stem cells into functional hepatic cells. Hepatology 45: 1229-1239. [PubMed]

57. Aurich H, Sgodda M, Kaltwasser P, Vetter M, Weise A, et al. (2009) Hepatocyte differentiation of mesenchymal stem cells from human adipose tissue in vitro promotes hepatic integration in vivo. Gut 58: 570-581. [PubMed]

58. Schwartz RE, Linehan JL, Painschab MS, Hu WS, Verfaillie CM, et al. (2005) Defined conditions for development of functional hepatic cells from human embryonic stem cells. Stem Cells Dev 14: 643-655. [PubMed]

59. Gouon-Evans V, Boussemart L, Gadue P, Nierhoff D, Koehler Cl, et al. (2006) BMP-4 is required for hepatic specification of mouse embryonic stem cellderived definitive endoderm. Nat Biotechnol 24: 1402-1411. [PubMed]

60. Touboul T, Hannan NR, Corbineau S, Martinez A, Martinet C, et al. (2010) Generation of functional hepatocytes from human embryonic stem cells under chemically defined conditions that recapitulate liver development. Hepatology 51: 1754-1765. [PubMed]

61. Duan Y, Ma X, Zou W, Wang C, Bahbahan IS, et al. (2010) Differentiation and characterization of metabolically functioning hepatocytes from human embryonic stem cells. Stem cells 28: 674-686. [PubMed]

62. Agarwal S, Holton KL, Lanza R (2008) Efficient differentiation of functional hepatocytes from human embryonic stem cells. Stem cells 26: 1117-1127. [PubMed]

63. Wojtczak A, Wojtczak M, Skretkowicz J (2014) The relationship between plasma concentration of metoprolol and CYP2D6 genotype in patients with ischemic heart disease. Pharmacol Rep 66: 511-514. [PubMed]

64. Bray J, Sludden J, Griffin MJ, Cole M, Verrill M, et al. (2010) Influence of pharmacogenetics on response and toxicity in breast cancer patients treated with doxorubicin and cyclophosphamide. $\mathrm{Br} \mathrm{J}$ Cancer 102: 1003-1009. [PubMed]

65. James LP, Simpson PM, Farrar HC, Kearns GL, Wasserman GS, et al. (2005) Cytokines and toxicity in acetaminophen overdose. J Clin Pharmacol 45: 11651171. [PubMed]

66. Prescott LF, Roscoe P, Wright N, Brown SS (1971) Plasma-paracetamol halflife and hepatic necrosis in patients with paracetamol overdosage. Lancet 1: 519-522. [PubMed]

67. Arkadopoulos N, Lilja H, Suh KS, Demetriou AA, Rozga J (1998) Intrasplenic transplantation of allogeneic hepatocytes prolongs survival in anhepatic rats. Hepatology 28: 1365-1370. [PubMed]

68. Lee KC, Palacios Jimenez C, Alibhai H, Chang YM, Leckie PJ, et al. (2013) A reproducible, clinically relevant, intensively managed, pig model of acute liver failure for testing of therapies aimed to prolong survival. Liver Int 33: 544-551. [PubMed] 